\title{
Clinical Characteristics of Patients with Oral Candidiasis
}

\author{
Ji Hoo Kim ${ }^{1,2}$, Jong-Mo Ahn ${ }^{2}$ \\ 1 Department of Dentistry, Graduate School, Chosun University, Gwangju, Korea \\ ${ }^{2}$ Department of Oral Medicine, School of Dentistry, Chosun University, Gwangju, Korea
}

Received May 10, 2021

Revised May 28, 2021

Accepted May 28, 2021
Correspondence to:

Jong-Mo Ahn

Department of Oral Medicine, School of Dentistry, Chosun University, 309 Pilmun-

daero, Dong-gu, Gwangju 61452, Korea

Tel: +82-62-220-3896

Fax: +82-62-234-2119

E-mail: jmahn@chosun.ac.kr

https://orcid.org/0000-0002-3615-3688

This study was supported by research fund from Chosun University Dental Hospital 2020.
Purpose: Oral candidiasis is the most common fungal infection in the oral cavity which is usually diagnosed from clinical findings. A retrospective study was conducted to identify risk factors for oral candidiasis and to characterize the demographic and clinical features of affected patients.

Methods: From January 1, 2019 to December 31, 2019, it consisted of 90 oral candidiasis patients diagnosed based on clinical finding and treated with antifungal drugs. As a retrospective study of those people, surveys were conducted on sex, age, systemic disease, a use of dentures, complaints of dry mouth, smoking and alcohol consumption, culture on potato dextrose agar (PDA) medium, culture on chromogenic agar (CA) medium and a duration of antifungal treatment.

Results: Among 90 selected patients, the male and female ratio was 41:49. Overall, female had a higher infection rate than male in all age groups. In this study, oral candidiasis was not clearly susceptible to dry mouth, smoking or drinking, wearing dentures and association with systemic disease. Among 90 patients with oral candidiasis, 83 had colonies formed on PDA medium and 53 had colonies formed on CA medium. The duration of antifungal treatment was highest between 5 and 8 weeks. In addition, there was statistical significance between the culture results in CA medium and the duration of antifungal treatment.

Conclusions: Generally, old age or infants, dry mouth, smoking, a use of dentures and endocrine abnormalities are risk factors to increase oral candidiasis; however, in this study, it was mainly found in the elderly aged 60 or older regardless of sex and the incidence of oral candidiasis was not obviously related with patients with dry mouth, smoking or drinking, denture wearers and endocrine abnormalities. Interestingly, when the fungi were cultured in CA medium, the duration of antifungal treatment was increased.

Key Words: Clinical characteristics; Oral candidiasis

\section{INTRODUCTION}

Oral candidiasis is the most common opportunistic infection affecting the oral mucosa. Most infections are caused by Candida albicans. Also, C. albicans, Candida tropicalis, Candida krusei and Candida glabrata are another reasons of infections [1,2]. Candida infection usually affects the lining of the oral mucosa, but in rare cases, it can be fatal when it affects the whole body. In order to invade the mucosal lining, microorganisms must adhere to the epithelial surface. Candida strains with better adhesion cause infection more easily than strains with low adhesion [1].

Traditionally, oral candidiasis can be divided into acute candidiasis and chronic candidiasis. There are pseudomembranous oral candidiasis and atrophic oral candidiasis in acute candidiasis, and hyperplastic oral candidiasis and atrophic oral candidiasis in chronic candidiasis. Pseudomembranous oral candidiasis includes thrush,

Copyright (c) 2021 Korean Academy of Orofacial Pain and Oral Medicine. All rights reserved.

(c) This is an open-access article distributed under the terms of the Creative Commons Attribution Non-Commercial License (http://creativecommons.org/licenses/by-nc/4.0/), which permits unrestricted non-commercial use, distribution, and reproduction in any medium, provided the original work is properly cited. 
atrophic oral candidiasis includes median rhomboid glossitis, antibiotic sore mouth, denture stomatitis and angular cheilitis, and hyperplastic oral candidiasis includes diseases such as Candida leukoplakia [3].

In some cases of oral candidiasis, symptoms may disappear spontaneously except for the cases of the use of antibiotics or topical corticosteroids. In other cases, treatment is sometimes necessary. There are treatment methods like the removal of trigger factors like smoking cessation, relief of dry mouth, improvement of oral hygiene, antifungal agents and etc. When using antifungal agents, topical antifungal agents are effective in treating all lesions confined to the oral cavity. Besides, the types of the medication are suspensions, tablets, creams and so on. The suspension is effective in patients with dry mouth who have difficulty dissolving the pills. Antifungal agents such as nystatin, amphotericin, miconazole, and fluconazole are sometimes used topically or systemically.

Although there are many studies and data on the related factors of oral candidiasis, large, single-centred retrospective studies are still not enough to identify common risk factors for oral candidiasis and to evaluate oral candidiasis compared to those identified in other studies [4-7]. Therefore, to characterize the clinical features of oral candidiasis, one-year retrospective study was conducted on patients diagnosed and treated with oral candidiasis who visited the Department of Oral Medicine at Chosun University Dental Hospital in Gwangju.

\section{MATERIALS AND METHODS}

Between January 1, 2019 and December 31, 2019, the clinical records of 90 patients diagnosed with oral candidiasis were reviewed in the clinical examination (Table 1) $[1,3,8]$ at the Department of Oral Medicine (hereinafter Oral Medicine) at Chosun University Dental Hospital in Gwangju. Data such as sex, age, general disease, a use of dentures, complaints of dry mouth, smoking or alcohol consumption, culture results in potato dextrose agar (PDA) and chromogenic agar (CA) medium, and duration of antifungal drug administration were collected. This study was conducted with the approval of the Clinical Ethics Review Committee of the Chosun University Dental Hospital (CUDHIRB-2101-001).

The diagnosis of oral candidiasis in this study was made according to the clinical features established in other studies based on clinical tests (Table 1) [1,3,8].

In this study, a total of 180 clinical specimens (2 for every 90 patients) were collected by rubbing the palatal mucosa and tongue of patients with a sterile cotton swab who visited the Oral medicine Department for culture of Candida strains. All specimens were plated on PDA medium (Potato Dextrose Agar; kisanBio Co., Ltd., Seoul, Korea) and CA medium (CHROMagar Candida; Chosun University Dental Hospital Department of Oral medicine, Gwang-ju, Korea). CA medium was obtained by purchasing Candida Chromogenic Agar powder (Condalab, Madrid, Spain), made by the Department of Oral Medicine at Chosun University Dental Hospital according to the manufacturer's

Table 1. The classification and characteristics of oral candidiasis for diagnosis

\begin{tabular}{|c|c|c|c|}
\hline Clinical type & Appearance and symptom & Common site & Associated factor \\
\hline Acute pseudomembranous & $\begin{array}{l}\text { Creamy-white plaques; removable; } \\
\text { burning sensation, foul taste }\end{array}$ & Buccal mucosa, tongue, palate & $\begin{array}{l}\text { Antibiotic therapy, } \\
\text { immunosuppression }\end{array}$ \\
\hline Acute or chronic atrophic & Red macules, burning sensation & $\begin{array}{l}\text { Posterior hard palate, buccal } \\
\text { mucosa, dorsal tongue }\end{array}$ & $\begin{array}{l}\text { Antibiotic therapy, xerostomia, } \\
\text { immunosuppression, idiopathic, } \\
\text { use of denture, loss of vertical } \\
\text { dimension }\end{array}$ \\
\hline Chronic hyperplastic & $\begin{array}{l}\text { White plaques that are not } \\
\text { removable; asymptomatic }\end{array}$ & Anterior buccal mucosa & $\begin{array}{l}\text { Idiopathic, immunosuppression; } \\
\text { care must be taken not confuse } \\
\text { this with keratotic lesions with } \\
\text { superimposed candidiasis }\end{array}$ \\
\hline
\end{tabular}

Data from the article of Glick et al. (Burket's oral medicine. 12th ed.) [1]; Korean Academy of Orofacial Pain and Oral Medicine (Diagnosis and treatment of oral soft tissue disease) [3]; and Reinhardt et al. (Braz Oral Res 2018:32:e92) [8]. 


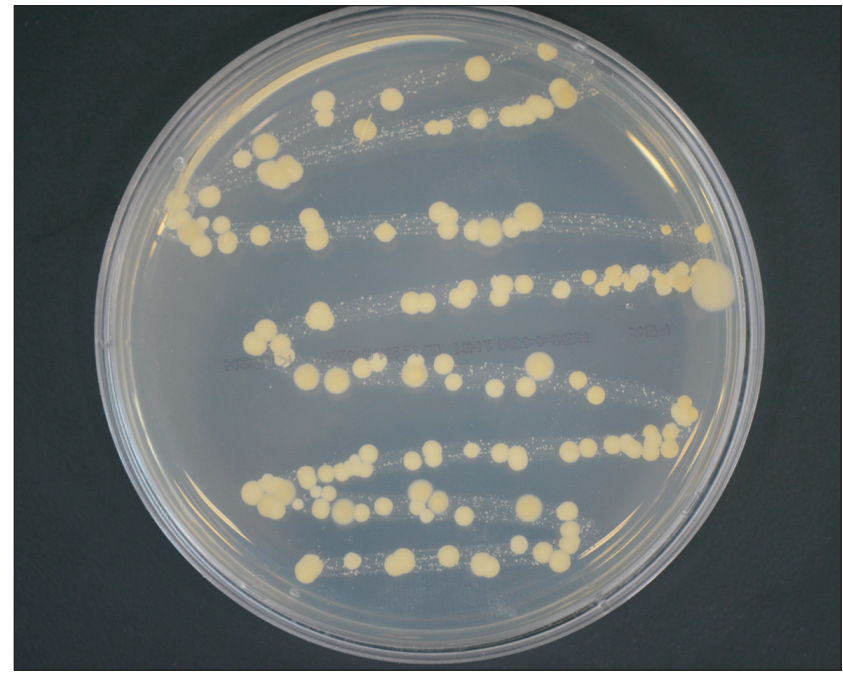

Fig. 1. Result of culture on potato dextrose agar (PDA) medium. This is the result of incubation at $37^{\circ} \mathrm{C}$ for 48 hours after rubbing the palatal mucosa and tongue of one patient with a sterile cotton swab and smearing it on PDA medium. If cream-colored colonies appeared, they were considered positive.

instructions, and distributed $20 \mathrm{~mL}$ each in a $60 \mathrm{~mm} \times 15$ $\mathrm{mm}$ petri dish. After smear, the medium was cultured for 48 hours in incubator (IN-20A) (Kukje Engineering Inc., Co., Ltd., Goyang, Korea) at $37^{\circ} \mathrm{C}$. On the PDA medium, all colonies were cream-colored, and the colonies' size and margin were varied (Fig. 1). Colonies in CA medium were confirmed to be cultured by pigmentation as described in the manufacturer's instructions and in Odds and Bernaerts (Fig. 2) [9]. In addition, when the number of colonies was observed 5 or less in the PDA medium and CA medium, it was determined as negative. In medical records, sex (male or female); age ( $<20$ years, $20-40$ years old, $40-60$ years old, $>60$ years old); presence of systemic disease (yes or no/World Health Organization International Classification of Disease-10, 2019); presence or absence of dentures (yes or no); presence of dry mouth (yes or no); smoking and alcohol consumption (yes or no); culture results in PDA and CA medium (yes or no); and duration of antifungal treatment (less than 4 weeks, more than 4 weeks to 8 weeks or less, more than 8 weeks to 12 weeks or less, more than 12 weeks to 16 weeks or less) were investigated. When examining the type of systemic disease, all cases with multiple systemic diseases were also investigated.

All statistical tests were performed by using Statistical Package for the Social Sciences (SPSS) software, version

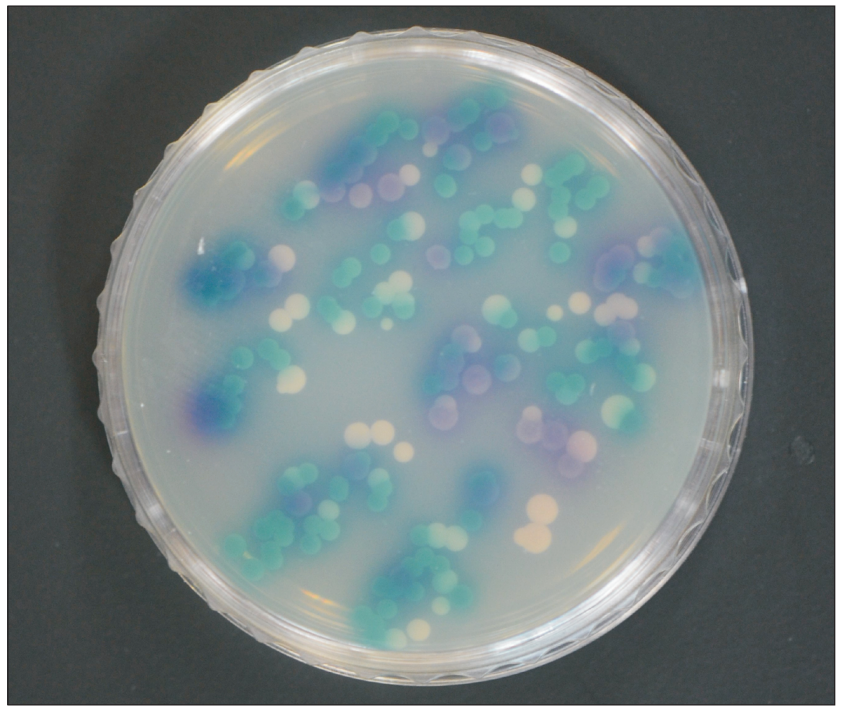

Fig. 2. Result of culture on chromogenic agar (CA) medium. This is the result of incubation at $37^{\circ} \mathrm{C}$ for 48 hours after rubbing the palatal mucosa and tongue of one patient with a sterile cotton swab and smearing it on CA medium. All four species can be distinguished by colony appearance: Candida albicans colonies are green or apple green, Candida glabrata are lavender, Candida tropicalis colonies are metallic blue, Candida parapsilosis colonies are pale cream.

22.0 (IBM Corp., Armonk, NY, USA), and the correlation of local factors with the duration of antifungal drug administration was analysed by using the Mann-Whitney $\mathrm{U}$ test and Kruskal-Wallis test at the 5\% significance level $(\mathrm{p}<0.05)$.

\section{RESULTS}

As for the male and female ratio, 41 males (45.6\%) and 49 females (54.4\%) were surveyed, with a large percentage of patients over 60s. Systemic diseases were not found in $11.9 \%$ of patients, and systemic diseases were investigated in the remaining patients based on the 10th revision of the International Classification of Diseases (ICD) (Table 2). As a result of surveying the number of each disease group and the percentage of all patients, 4 patients with blood and blood forming organs diseases and certain disorders involving the immune mechanism (2.4\%), 37 patients with endocrine, nutritional and metabolic diseases (22.0\%), 11 patients with mental and behavioural disorders (6.5\%), 8 patients with nervous system diseases (4.8\%), 2 patients with eye and appendage diseases (1.2\%), 9 patients with circulatory system diseases (20.8\%), 6 patients respiratory system 
Table 2. The distribution and ratio of systemic disease by ICD-10

\begin{tabular}{|c|c|c|}
\hline Code & Systemic diseases by ICD-10: 2019 & Patient (\%) \\
\hline $\mathrm{N} / \mathrm{S}$ & Non-specific symptoms and signs & $20(11.9)$ \\
\hline I & Certain infectious and parasitic diseases & 0 \\
\hline II & Neoplasms & 0 \\
\hline III & Diseases of the blood and blood-forming organs and certain disorders involving the immune mechanism & $4(2.4)$ \\
\hline IV & Endocrine, nutritional and metabolic diseases & $37(22.0)$ \\
\hline $\mathrm{V}$ & Mental and behavioural disorders & $11(6.5)$ \\
\hline VI & Diseases of the nervous system & $8(4.8)$ \\
\hline VII & Diseases of the eye and adnexa & $2(1.2)$ \\
\hline VIII & Diseases of the ear and mastoid process & 0 \\
\hline IX & Diseases of the circulatory system & $9(20.8)$ \\
\hline $\mathrm{x}$ & Diseases of the respiratory system & $6(3.6)$ \\
\hline $\mathrm{XI}$ & Diseases of the digestive system & $14(8.3)$ \\
\hline XII & Diseases of the skin and subcutaneous tissue & $4(2.4)$ \\
\hline XIII & Diseases of the musculoskeletal system and connective tissue & $16(9.5)$ \\
\hline XIV & Diseases of the genitourinary system & $10(6.0)$ \\
\hline $\mathrm{XV}$ & Pregnancy, childbirth and the puerperium & $1(0.6)$ \\
\hline
\end{tabular}

ICD, International Classification of Diseases; N/S, non-specific.

Values are presented as number (\%).

disease (3.6\%), 14 patients with digestive system disease (8.3\%), 4 patients with skin and subcutaneous tissue disease (2.4\%), 16 patients with musculoskeletal and connective tissue diseases (9.5\%), 10 patients with urogenital diseases (6.0\%), and 1 patient with pregnancy, childbirth and delivery $(0.6 \%)$, of which endocrine, nutrition and metabolic diseases accounted for the highest proportion (Table 2). Also, $25.6 \%$ of patients used dentures, $44.4 \%$ of patients complained of dry mouth, and 8.9\% patients were a smoker or an alcohol consumer (Table 3).

As a result of smears by using sterile cotton swabs in the oral cavity of 90 patients with oral candidiasis, 83 had colonies formed on PDA medium (92.2\%) and 53 had colonies formed on CA medium (58.9\%) (Table 3). For oral candidiasis patients, Diflucan dry syrup $350 \mathrm{mg} / 35 \mathrm{~mL}$ [10 mg/mL] (Pfizer Pharmaceutical Korea Ltd., Seoul, Korea) was applied topically in the oral cavity at $50 \mathrm{mg}$ per day for treatment. The duration of drug administration was high between 5 and 8 weeks (Table 3).

Statistical analysis was performed by using the MannWhitney U test and Kruskal-Wallis at the 5\% significance level, which was for local factors of the duration of antifungal drug administration. As a result, there was statistical significance between the culture results of CA medium and the duration of antifungal treatment ( $\mathrm{p}=0.012$; Table 3$)$.

\section{DISCUSSION}

Oral candidiasis is usually diagnosed through visual examination of removable white plaque or erythema tissue in the oral cavity, and microscopic examination of a sample of the oral mucosa with characteristic findings. Also, diagnosis of oral candidiasis can be based on clinical and microbiological test [8]. To reach an accurate diagnosis, it is an essential to make a balance between clinical findings and laboratory data; meanwhile, sometimes antifungal therapy would be initiated to aid the diagnosis process [1]

The prevalence of Candida strains which are part of the oral flora is diverse geographically, but has been reported in several studies to be average 35\% [1]. The presence of a fungus simply does not indicate an infection [8]. Also, the incidence rate varies with age and certain predisposing factors [10]. Oral candidiasis affects a large part of the population, especially children and the elderly, and is considered an opportunistic infection that occurs more often in people with weakened immunity [8].

Candida strains are more often isolated from the oral mucosa of women [1], and become more sensitive to external stimuli with aging [11]. In this study, women developed oral candidiasis more frequently than men, but there was no significant difference, and oral candidiasis was more common among women over 60 years of age. A study by Reinhardt 
Table 3. Results of investigation by each local factors and statistical analysis

\begin{tabular}{|c|c|c|c|}
\hline Local factor & n (\%) & $95 \% \mathrm{Cl}$ & $\mathrm{p}$-value \\
\hline Sex & & & $0.140^{\mathrm{a}}$ \\
\hline Female & 49 (54.4) & $3.45(2.80-4.10)$ & \\
\hline Male & $41(45.6)$ & $4.51(3.63-5.49)$ & \\
\hline Age $(y)$ & & & $0.147^{b}$ \\
\hline$<20$ & $1(1.1)$ & 13 & \\
\hline $20-40$ & $5(5.6)$ & $2.60(1.00-5.80)$ & \\
\hline $40-60$ & $19(21.1)$ & $3.53(2.68-4.37)$ & \\
\hline$>60$ & $65(72.2)$ & $4.02(3.34-4.75)$ & \\
\hline Denture wearer & & & $0.096^{\mathrm{a}}$ \\
\hline No & $67(74.4)$ & $3.69(3.03-4.40)$ & \\
\hline Yes & $23(25.6)$ & $4.65(3.61-5.70)$ & \\
\hline Complaints of dry mouth & & & $0.240^{\mathrm{a}}$ \\
\hline No & $50(55.6)$ & $4.20(3.40-4.92)$ & \\
\hline Yes & $40(44.4)$ & $3.60(2.80-4.60)$ & \\
\hline Smoking and drinking & & & $0.102^{b}$ \\
\hline No & $56(62.2)$ & $4.07(3.36-4.79)$ & \\
\hline Only smoking & $6(6.7)$ & $6.33(5.00-9.00)$ & \\
\hline Only drinking & $20(22.2)$ & $3.40(2.40-4.40)$ & \\
\hline Both yes & $8(8.9)$ & $2.50(1.50-4.00)$ & \\
\hline Culture on PDA medium & & & $0.474^{\mathrm{a}}$ \\
\hline No & $7(7.8)$ & $3.29(1.57-5.57)$ & \\
\hline Yes & $83(92.2)$ & $3.99(3.41-4.66)$ & \\
\hline Culture on CA medium & & & $0.012^{\mathrm{a} *}$ \\
\hline No & $37(41.1)$ & $3.16(2.30-4.14)$ & \\
\hline Yes & $53(58.9)$ & $4.47(3.72-5.15)$ & \\
\hline Duration of antifungal treatment (wk) & & & - \\
\hline$\leq 4$ & $36(40.0)$ & - & \\
\hline $5-8$ & $44(48.9)$ & - & \\
\hline $9-12$ & $8(8.9)$ & - & \\
\hline $13-16$ & $2(2.2)$ & - & \\
\hline
\end{tabular}

$\mathrm{Cl}$, confidence interval; PDA, potato dextrose agar; CA, chromogenic agar; -, not available.

${ }^{a}$ Statistical significance was evaluated using the Mann - Whitney $U$ test.

${ }^{b}$ Statistical significance was evaluated using the Kruskal - Wallis test.

*There was statistical significance $(p<0.05)$ between the culture results of CA medium and the administration period of the antifungal agent.

et al. [8] also found that women are more often affected by oral candidiasis than men, and women are more likely to be treated for symptoms of oral candidiasis and the incidence rate increases with age. In addition, it is known that older people, especially those who have difficulty in oral hygiene and a use of dentures, have a higher incidence of the disease $[2,8,10]$.

The oral mucosa is likely to be damaged by acquired systemic diseases, and candidiasis is often reported to be associated with systemic diseases (e.g. diabetes mellitus, cardiovascular disease, depression, immune suppression) [12]. However, the relationship between the presence of systemic disease and oral candidiasis is unclear according to literature evidence [8]. Systemic factors such as diabetes mellitus, immunosuppression, malignancies, malnutrition, and use of antibiotics, or diseases such as stomatitis, dry mouth, and pemphigus are known to be very susceptible to oral candidiasis [2,8]. In this study, more than $80 \%$ of patients diagnosed with oral candidiasis had at least one systemic disease. Among them, it is believed that the endocrine, nutritional and metabolic disease groups show a high proportion, and it is estimated that the presence of systemic conditions such as diabetes mellitus and malnutrition may have been more susceptible to candida infection.

Oral candidiasis is said to be particularly common disease in people who wear dentures [8], but the proportion of patients diagnosed with oral candidiasis in this study was 25.6\%. According to an epidemiologic study by Gendreau 
and Loewy [13], the prevalence of denture stomatitis among denture wearers was $15 \%$ to $70 \%$. In cases reported by Gendreau and Loewy [13], the incidence of denture stomatitis is higher in elderly denture wearers and women, and there are etiological factors including poor dental hygiene, continuous use and nighttime use of removable dentures, plaque accumulation, bacteria and yeast contamination. Furthermore, if the denture doesn't fit, it can cause mucosal trauma. All of these factors appear to increase the ability of C. albicans to colonize both the denture and oral mucosa surfaces. Antifungal therapy can eradicate $C$. albicans contamination and relieve symptoms of stomatitis; however, stomatitis recurs when antifungal therapy is stopped unless the dentures are kept clean [13]. Denture hygiene is vital for removing nutrients, including exfoliated epithelial cells, which can serve as an essential nitrogen source for yeast growth. In addition, denture cleaning interferes with the maturity of the microorganism environment established under the denture. Denture should be stored in an antibacterial solution at night as there may be microorganisms on the porous surface of the denture that cannot be removed by physical cleaning. A variety of solutions are used including alkaline peroxide, alkaline hypochlorite, acids, disinfectants and enzymes [1].

Saliva plays a central role in maintaining oral homeostasis, function and health. The prevalence and consequences of dry mouth are increasing due to the aging, growing population, the effects of some systemic diseases, medications and drugs that reduce saliva production [14]. According to Ohga et al. [15], dry mouth is mainly caused by decreased saliva secretion and is known to be a high-risk factor for oral candidiasis [15,16]. According to Torres et al. [17], the signs and symptoms of dry mouth are closely related to changes in the oral microbial community. A decrease in the stimulated total salivary flow rate (SWS) can alter the oral microflora, thus increasing the risk of oral candidiasis [17]. However, in this study, 44.4\% of patients with oral candidiasis complained of dry mouth, but it was difficult to prove the association between dry mouth and oral candidiasis infection.

Smoking is a factor that can induce erythematous oral candidiasis. In particular, there is a high risk of developing median rhomboid glossitis. If one quit smoking, Candida infection could disappear without antifungal therapy $[1,10]$. The components of tobacco smoke can cause chronic inflammation in the oral mucosa, impair innate immune mechanisms against pathogens, and inhibit cell growth through apoptosis mechanisms. The smoking reduces the production of salivary enzymes and immunoglobulins, and affects lymphocyte production, resulting in microscopic imbalance of the oral cavity [18]. According to the Weinstein et al.'s study [19], there is no difference between smokers and non-smoker samples in bacterial composition, but median rhomboid glossitis, chronic hyperplastic candidiasis, and other diseases were associated with smoking [19]. Drinking alcohol is a contributing factor to oral candidiasis, and frequent drinking is considered a risk factor for developing oral candidiasis. C. albicans induces high levels of acetaldehyde production by oxidizing saliva ethanol, which acetaldehyde increases permeability of the oral mucosa. Furthermore, this acetaldehyde affects the oral mucosa, causing atrophic regions on the epithelial surface that lacks extracellular lipids due to alcohol consumption. In addition, a glucose concentration of $18 \mathrm{~g} / \mathrm{dL}$, which can be easily obtained from alcohol, promotes the occurrence of oral candidiasis by increasing biofilm formation and C. albicans adhesion [20]. In this study, 8.9\% of those who did smoke and drink alcohol were lower, but in Gonçalves et al. [21] and Epstein et al. [22]'s studies, an important association was observed between oral candidiasis and smoking or alcohol consumption.

PDA medium can be used for yeast and fungal culture and counting in food and dairy products. Among microorganisms, Aspergillus niger, C. albicans, and Saccharomyces cerevisiae can grow. We could not find any details on the sensitivity or specificity of the PDA medium. However, in an experiment by Madhavan et al. [23], when comparing the results of candida culture in CA medium with candida culture in PDA medium, all 7 species studied (C. albicans, Candida dubliniensis, C. tropicalis, C. glabrata, Candida rugosa, C. krusei and Candida parapsilosis) appeared as cream and found no difference in colony color. Other characteristics, such as colony size, margins, and height, vary by species. These colony characteristics were the same as observed using CA medium [23].

CA medium is a differential culture medium that allows 
selective isolation of yeast and at the same time identifies colonies of $C$. albicans, $C$. tropicalis and $C$. krusei. It is technically simple and fast, and cost of testing is low compared to the conventional methods [24]. More than 95\% of clinical seperation of $C$. albicans, C. tropicalis and C. krusei were correctly identified based on colony morphology and pigmentation in CA medium [24], providing reliable and rapid identification of the most common $C$. albicans, and presumptive identification of $C$. tropicalis and $C$. krusei [25]. In addition, according to the experimental results of Madhavan et al. [23], the sensitivity of the CA medium to identify C. albicans, C. dubliniensis, C. tropicalis, C. glabrata, $C$. rugosa, $C$. krusei and C. parapsilosis ranged between 25 and $100 \%$ at $30^{\circ} \mathrm{C}, 14 \%$ and $100 \%$ at $35^{\circ} \mathrm{C}, 56 \%$ and $100 \%$ at $37^{\circ} \mathrm{C}$. The specificity of the CA medium was $100 \%$ at $30^{\circ} \mathrm{C}$, between $97 \%$ and $100 \%$ at $35^{\circ} \mathrm{C}, 92 \%$ and $100 \%$ at $37^{\circ} \mathrm{C}$ [23]. In this study, 92,2\% were cultured in PDA medium and 58.9\% in CA medium. It shows a statistically significant difference between the culture results of CA medium and the duration of antifungal treatment $(\mathrm{p}=0.012$; Table 3$)$. In other words, when colony formation is observed in the CA medium, which is a Candida selective medium, it means that the administration period of the antifungal agent is prolonged. Also, the culture result of the CA medium can be considered when prescribing an antifungal agent.

There are several drugs to treat oral candidiasis. Although Nystatin and Miconazole are the most commonly used topical antifungal agents and are very effective, they take a long time to eradicate the infection. Miconazole is well tolerated, but may interact with other drugs. Therefore, it should be considered before using the drug. Besides, amphotericin B or clotrimazole is not available in many countries. Nystatin and fluconazole are very effective in treating oral candidiasis. Especially, oral fluconazole use is effective in treating oral candidiasis that does not respond to topical treatment [26]. As a result of the meta-analysis of Lyu et al. [27], nystatin pills were significantly superior to placebo in the treatment of denture stomatitis; however, nystatin suspension was not superior to fluconazole in the treatment of oral candidiasis in infants, children, or human immunodeficiency virus/acquired immune deficiency syndrome patients [27].
Fluconazole is an excellent triazole line for the treatment of candida infections including oropharyngeal and esophageal candidiasis, vulvovaginal candidiasis and disseminated candidiasis. It is an oral and parenteral fungicide that inhibits the synthesis of ergosterol in yeast. Extensive clinical studies by Cha and Sobel [28] demonstrate fluconazole's surprising efficacy, favorable pharmacokinetics and a reliable safety profile. In a study by Epstein et al. [29], 10 women and 9 men diagnosed with oral candidiasis used fluconazole $(2 \mathrm{mg} / \mathrm{mL})$ aqueous solution three times a day as a topical treatment by rinsing and spitting for 1 week. As a result of this treatment, complete symptoms and clinical relief in $94 \%$ of patients was noted, fungal treatment was demonstrated in patients except for only one patient, and no side effects were reported [29]. Fluconazole was also shown to be superior to nystatin suspension in the treatment of pseudomembranous candidiasis in healthy infants [30]. Also, oral rinsing with fluconazole suspensions may be useful in managing dry mouth patients or patients with difficulty swallowing due to oral candidiasis [29]. Hence, in this study, we selected a method of topically applying Diflucan dry syrup $350 \mathrm{mg} / 35 \mathrm{~mL}$ [10 mg/mL] to oral candidiasis patients by rinsing the oral cavity of $50 \mathrm{mg}$ daily for a minimum of 2 weeks to a maximum of 16 weeks.

In this study, objective data to support the diagnosis such as inter-observer or intra-observer reliability were lacking in the diagnosis based on clinical features of oral candidiasis. Patients complaining of dry mouth could not be confirmed using test methods such as saliva secretion. In addition, this study had its own limitations in that it was not possible to perform large sample sizes, candida species identification, and antifungal susceptibility tests. However, CA medium proved to be a valuable method of identifying Candida species even in resource-poor environments, and it was found that the duration of antifungal treatment was also relevant.

\section{CONFLICT OF INTEREST}

No potential conflict of interest relevant to this article was reported. 


\section{ORCID}

\author{
Ji Hoo Kim \\ https://orcid.org/0000-0002-0669-3309 \\ Jong-Mo Ahn \\ https://orcid.org/0000-0002-3615-3688
}

\section{REFERENCES}

1. Glick M, Feagans WM. Burket's oral medicine. 12th ed. Shelton: People's Medical Publishing House; 2015. pp. 93-99.

2. Hu L, He C, Zhao C, Chen X, Hua H, Yan Z. Characterization of oral candidiasis and the Candida species profile in patients with oral mucosal diseases. Microb Pathog 2019;134:103575.

3. Korean Academy of Orofacial Pain and Oral Medicine. Diagnosis and treatment of oral soft tissue disease. Seoul: Shinhung International; 2010. pp. 274-281.

4. Kossioni AE. The prevalence of denture stomatitis and its predisposing conditions in an older Greek population. Gerodontology 2011;28:85-90.

5. Mima EG, Vergani CE, Machado AL, et al. Comparison of Photodynamic Therapy versus conventional antifungal therapy for the treatment of denture stomatitis: a randomized clinical trial. Clin Microbiol Infect 2012;18:E380-E388.

6. Maciąg J, Osmenda G, Nowakowski D, et al. Denture-related stomatitis is associated with endothelial dysfunction. Biomed Res Int 2014;2014:474016.

7. Tay LY, Jorge JH, Herrera DR, Campanha NH, Gomes BP, Andre Dos Santos F. Evaluation of different treatment methods against denture stomatitis: a randomized clinical study. Oral Surg Oral Med Oral Pathol Oral Radiol 2014;118:72-77.

8. Reinhardt LC, Nascente PDS, Ribeiro JS, Etges A, Lund RG. A single-center 18-year experience with oral candidiasis in Brazil: a retrospective study of 1,534 cases. Braz Oral Res 2018;32:e92.

9. Odds FC, Bernaerts R. CHROMagar Candida, a new differential isolation medium for presumptive identification of clinically important Candida species. J Clin Microbiol 1994;32:1923-1929.

10. Akpan A, Morgan R. Oral candidiasis. Postgrad Med J 2002;78: 455-459.

11. Saintrain MV, Holanda TG, Bezerra TM, de Almeida PC. Prevalence of soft tissue oral lesion in elderly and its relations with deleterious habits. Gerodontology 2012;29:130-134.

12. Semlali A, Killer K, Alanazi H, Chmielewski W, Rouabhia M. Cigarette smoke condensate increases C. albicans adhesion, growth, biofilm formation, and EAP1, HWP1 and SAP2 gene expression. BMC Microbiol 2014;14:61.

13. Gendreau L, Loewy ZG. Epidemiology and etiology of denture stomatitis. J Prosthodont 2011;20:251-260.

14. Anil S, Vellappally S, Hashem M, Preethanath RS, Patil S, Samaranayake LP. Xerostomia in geriatric patients: a burgeoning global concern. J Investig Clin Dent 2016;7:5-12.
15. Ohga N, Yamazaki Y, Sato J, et al. Elimination of oral candidiasis may increase stimulated whole salivary flow rate. Arch Oral Biol 2016;71:129-133.

16. Billings M, Dye BA, Iafolla T, Grisius M, Alevizos I. Elucidating the role of hyposalivation and autoimmunity in oral candidiasis. Oral Dis 2017;23:387-394.

17. Torres SR, Peixoto CB, Caldas DM, et al. Relationship between salivary flow rates and Candida counts in subjects with xerostomia. Oral Surg Oral Med Oral Pathol Oral Radiol Endod 2002;93:149-154.

18. Lee J, Taneja V, Vassallo R. Cigarette smoking and inflammation: cellular and molecular mechanisms. J Dent Res 2012;91:142-149.

19. Weinstein RL, Francetti L, Maggiore E, Marchesi G. [Alcohol and smoking. The risk factors for the oral cavity]. Minerva Stomatol 1996;45:405-413. Italian.

20. Uittamo J, Siikala E, Kaihovaara P, Salaspuro M, Rautemaa R. Chronic candidosis and oral cancer in APECED-patients: production of carcinogenic acetaldehyde from glucose and ethanol by Candida albicans. Int J Cancer 2009;124:754-756.

21. Gonçalves LS, Júnior AS, Ferreira SM, et al. Factors associated with specific clinical forms of oral candidiasis in HIV-infected Brazilian adults. Arch Oral Biol 2013;58:657-663.

22. Epstein JB, Freilich MM, Le ND. Risk factors for oropharyngeal candidiasis in patients who receive radiation therapy for malignant conditions of the head and neck. Oral Surg Oral Med Oral Pathol 1993;76:169-174.

23. Madhavan P, Jamal F, Chong PP, Ng KP. Identification of local clinical Candida isolates using CHROMagar Candida ${ }^{\mathrm{TM}}$ as a primary identification method for various Candida species. Trop Biomed 2011;28:269-274.

24. Pfaller MA, Houston A, Coffmann S. Application of CHROMagar Candida for rapid screening of clinical specimens for Candida albicans, Candida tropicalis, Candida krusei, and Candida (Torulopsis) glabrata. J Clin Microbiol 1996;34:58-61.

25. Houang ET, Chu KC, Koehler AP, Cheng AF. Use of CHROMagar Candida for genital specimens in the diagnostic laboratory. J Clin Pathol 1997;50:563-565.

26. Quindós G, Gil-Alonso S, Marcos-Arias C, et al. Therapeutic tools for oral candidiasis: current and new antifungal drugs. Med Oral Patol Oral Cir Bucal 2019;24:e172-e180.

27. Lyu X, Zhao C, Yan ZM, Hua H. Efficacy of nystatin for the treatment of oral candidiasis: a systematic review and meta-analysis. Drug Des Devel Ther 2016;10:1161-1171.

28. Cha R, Sobel JD. Fluconazole for the treatment of candidiasis: 15 years experience. Expert Rev Anti Infect Ther 2004;2:357-366.

29. Epstein JB, Gorsky M, Caldwell J. Fluconazole mouthrinses for oral candidiasis in postirradiation, transplant, and other patients. Oral Surg Oral Med Oral Pathol Oral Radiol Endod 2002;93:671675.

30. Goins RA, Ascher D, Waecker N, Arnold J, Moorefield E. Comparison of fluconazole and nystatin oral suspensions for treatment of oral candidiasis in infants. Pediatr Infect Dis J 2002;21:11651167. 